\title{
Dynamical behaviors of a discrete SIS epidemic model with standard incidence and stage structure
}

\section{Zhidong Teng, Linfei Nie* and Jiabo Xu}

"Correspondence: Ifnie@163.com College of Mathematics and System Sciences, Xinjiang University, Urumqi, Xinjiang 830046, P.R. China

\begin{abstract}
A discrete SIS epidemic model with stage structure and standard incident rate which is governed by Beverton-Holt type is studied. The sufficient conditions on the permanence and extinction of disease are established. The existence of the endemic equilibrium is obtained. Further, by using the method of linearization, the local asymptotical stability of the endemic equilibrium is also studied. Lastly, the examples and numerical simulations carried out to illustrate the feasibility of the main results and revealed the far richer dynamical behaviors of the discrete epidemic model compared with the corresponding continuous epidemic models.
\end{abstract}

MSC: 39A30;92D30

Keywords: discrete SIS epidemic model; stage structure; permanence and extinction; endemic equilibrium; local stability

\section{Introduction}

Epidemics play an important role in the development and survival of human species. Recently, mathematical epidemic models in discrete time have been established to describe the dynamical evolution of epidemics. For example, in [1], Castillo-Chavez and Yakubu proposed a discrete SIS epidemic model and investigated its complex dynamics. In [2], some SI, SIR and SIS type epidemic models in discrete time are studied by Allen. In addition, many other results on the discrete time epidemic dynamical models can be seen in [3-16].

In the standard SIS model, the incidence rate is bilinear and is given by $\beta I S$, where $I$ and $S$ are respectively the numbers of infective and susceptible individuals per unit area and $\beta$ is the transmission rate. When $N$, the number of humans per unit area, is large, the adequate contact rate $\beta N$ which proportionates to $N$ is unreasonable, because the number of infected individuals which a susceptible individual contacts is limited. Anderson and May in [17] point out that standard incidence $\beta I S / N$ are more reasonable than bilinear incidence. Capasso and Serio in [18] introduced a saturated incidence rate $g(I)=k I /(1+\alpha I)$ into epidemic models in studying the cholera spread in Bari in Italy, where $k$ and $\alpha$ are positive constants. The general incidence rate $g(I)=k I^{q} /\left(1+\alpha I^{p}\right)$ was proposed by Liu, Levin and Iwasa in [19]; here, $q$ and $p$ are positive integers.

It is well known that many kinds of diseases, such as AIDS, SARS and tuberculosis, more easily spread among mature individuals. Thus, it is meaningful to consider models with 
stage structure. Stage structures were introduced in some continuous models (see, e.g., Xiao and Chen [20] and the references cited therein). However, up to now, there have been few results about discrete epidemic models with stage structure. In [3], a discrete epidemic model with stage structure governed by bilinear incidence are studied by $\mathrm{Li}$ and Wang. In this paper, we will investigate a discrete epidemic model with standard incidence and with disease that spreads only among mature individuals.

According to the transmission mechanism of some diseases, the population is divided into three classes: immature individuals, susceptible mature individuals and infectious mature individuals. We denote that $J(n)$ is the density of immature individuals, $S(n)$ is the density of the mature individuals, $I(n)$ is the density of infectious individuals and $N(n)=J(n)+S(n)+I(n)$ is the density of the total population. We assume that the susceptible individual becomes infectious after contact with infective individuals, and recovery from disease does not give permanent immunity. Furthermore, we suppose that the infective individual and the immature individuals have no ability to breed, which is reasonable, for example, women who are infected with gonorrhea cannot give birth to babies. Meanwhile, we suppose that the recruitment rate is governed by the Beverton-Holt type, that is, $\xi S(n) /(1+\beta N(n))$ and that susceptible individuals are infected with probability $\alpha I(n) / N(n)$.

Under the above assumptions, the following discrete SIS model with stage structure and standard incidence rate is proposed

$$
\left\{\begin{array}{l}
J(n+1)=\frac{\xi S(n)}{1+\beta N(n)}+r_{1} J(n)-r_{1} c J(n) \\
S(n+1)=r_{1} c J(n)+r_{2}\left(1-\frac{\alpha I(n)}{N(n)}\right) S(n)+r_{3} \sigma I(n), \\
I(n+1)=r_{2} \alpha \frac{I(n)}{N(n)} S(n)+r_{3}(1-\sigma) I(n) \\
N(n)=J(n)+S(n)+I(n)
\end{array}\right.
$$

where $\xi$ and $\beta$ are positive constants, $r_{1}$ is the survival rate of immature individuals, $r_{2}$ is the survival rate of mature individuals, $r_{3}$ is the survival rate of infected individuals, $c$ is the rate of immature individuals becoming mature individuals and $\sigma$ is the susceptible individuals that recover with probability.

For an epidemic model, it is well known that we are concerned about that whether the disease will invade the population. Thus, it is important to research the permanence and extinction of the disease. Therefore, in this paper, we firstly will establish the sufficient conditions, which ensure the disease in model (1) is permanent or extinct, respectively. Next, by using the Horn fixed-point theorem and the method of linearization, we will study the existence and local asymptotical stability of the endemic equilibrium for model (1). Further, for a discrete epidemic model, its dynamical behaviors may be very complicated which can be seen in some examples and numerical simulations in model (1).

The organization of this paper is as follows. In the next section, we will introduce the basic assumptions for model (1). Next, we will consider an auxiliary system, which includes a variable parameter (see model (7) in the next section). We will establish the sufficient conditions which ensure the global asymptotical stability of positive equilibrium of the auxiliary system. From this, we will further obtain the global asymptotical stability of positive equilibrium of the disease-free subsystem of model (1) (see model (3) in the next section). Further, we will consider the general autonomous difference equation (see 
model (9) in the next section). We will prove that if all solutions of the equation are ultimately bounded then it has at least an equilibrium. In Section 3, the sufficient conditions are established to ensure the permanence or extinction of the disease in model (1). Further, the existence and local asymptotical stability of endemic equilibrium are obtained in Section 4. In Section 5, some examples and numerical simulations are given to illustrate the feasibility of the main results. In Section 6, a brief discussion and some open problems are given.

\section{Preliminaries}

Let $Z$ denote the set of all nonnegative integers. In this paper, for model (1) we first introduce the following assumptions:

$\left(\mathrm{H}_{1}\right) \quad 0<c<1,0<\alpha<1,0<\sigma<1,0<r_{i}<1(i=1,2,3)$.

$\left(\mathrm{H}_{2}\right) \xi+R>1$, where $R=\max \left\{r_{1}, r_{2}, r_{3}\right\}$.

$\left(\mathrm{H}_{3}\right) r_{1}-r_{1} c-\xi / 4>0$.

Based on the biological background of model (1), we only consider the solution of model (1) with the following initial conditions:

$$
(J(0), S(0), I(0)) \in R_{+}^{3}=\{(x, y, z): x>0, y>0, z>0\} .
$$

Lemma 2.1 Assume that $\left(\mathrm{H}_{1}\right)$ holds. Then $R_{+}^{3}$ is the invariable set of model (1), that is, any solution $(J(n), S(n), I(n))$ of model (1) with initial condition (2) is positive for any $n \in Z$.

The proof of Lemma 2.1 is simple; we hence omit it here. When $I(n) \equiv 0$ in model (1), we obtain the following subsystem of model (1)

$$
\left\{\begin{array}{l}
J(n+1)=\frac{\xi S(n)}{1+\beta N(n)}+r_{1} J(n)-r_{1} c J(n) \\
S(n+1)=r_{1} c J(n)+r_{2} S(n) \\
N(n)=J(n)+S(n)
\end{array}\right.
$$

We firstly calculate equilibria of model (3). Considering the equation as follows

$$
\left\{\begin{array}{l}
J=\frac{\xi S}{1+\beta(J+S)}+r_{1} J-r_{1} c J, \\
S=r_{1} c J+r_{2} S
\end{array}\right.
$$

let

$$
A^{*}=\frac{\xi r_{1} c}{\left(1-r_{1}+r_{1} c\right)\left(1-r_{2}\right)} \text {. }
$$

Solving equation (4), we can obtain that if $A^{\prime \prime} \leq 1$ then equation (4) only has a solution $J=0, S=0$, and if $A^{\prime \prime}>1$ then equation (4) has two solutions $J=0, S=0$ and $J=J^{\prime \prime}, S=S^{*}$, where

$$
J^{*}=\frac{\left(A^{*}-1\right)\left(1-r_{2}\right)}{\left(1-r_{2}+r_{1} c\right) \beta}, \quad S^{*}=\frac{r_{1} c J^{*}}{\left(1-r_{2}\right)}
$$


Therefore, we finally have that if $A^{*} \leq 1$, then model (3) has only an equilibrium $E_{0}(0,0)$ and if $A^{*}>1$, then model (3) has two equilibria $E_{0}(0,0)$ and $E^{*}\left(J^{*}, S^{*}\right)$.

Corresponding to $E^{\prime \prime}\left(U^{*}, S^{*}\right)$, we see that model (1) has a disease-free equilibrium $\left(J^{\prime \prime}, S^{\prime \prime}, 0\right)$. Further, we introduce the following assumption:

$$
\operatorname{det}\left(\begin{array}{cc}
1-r_{1}+r_{1} c+\frac{\xi \beta S^{*}}{\left.\left(1+\beta U^{*}+S^{*}\right)\right)^{2}} & -\frac{\xi\left(1+\beta J^{*}\right)}{\left.\left(1+\beta U^{*}+S^{*}\right)\right)^{2}} \\
-r_{1} c & 1-r_{2}
\end{array}\right) \neq 0 .
$$

In order to study the extinction and permanence of the disease of model (1), we introduce an auxiliary system as follows:

$$
\left\{\begin{array}{l}
J(n+1)=\frac{\xi S(n)}{1+\beta(U(n)+S(n)+\rho)}+r_{1} J(n)-r_{1} c J(n), \\
S(n+1)=r_{1} c J(n)+r_{2}\left(1-\frac{\alpha \rho}{U(n)+S(n)+\rho)}\right) S(n),
\end{array}\right.
$$

where $\rho$ is a nonnegative parameter. Let

$$
B^{*}=\frac{\xi r_{1} c}{\left(1-r_{1}+r_{1} c\right)\left(1-r_{2}(1-\alpha)\right)} .
$$

We have the following result.

Lemma 2.2 Assume that $\left(\mathrm{H}_{1}\right)$ to $\left(\mathrm{H}_{4}\right)$ hold and $B^{*}>1$. Then there exists a positive constant $\delta>0$ such that model (7) has a globally uniformly asymptotically stable positive equilibrium $E_{\rho}^{*}\left(J_{\rho}^{*}, S_{\rho}^{*}\right)$ for any $\rho \in[0, \delta)$. Furthermore,

$$
\lim _{\rho \rightarrow 0} E_{\rho}^{*}\left(J_{\rho}^{*}, S_{\rho}^{*}\right)=E^{*}\left(J^{*}, S^{*}\right)
$$

Proof To obtain equilibria of model (7), we first consider the following equations:

$$
\left\{\begin{array}{l}
F(\rho, J, S)=J-\frac{\xi S}{1+\beta(U+S+\rho)}-r_{1} J+r_{1} c J=0, \\
G(\rho, J, S)=S-r_{1} c J-r_{2}\left(1-\frac{\alpha \rho}{(J+S+\rho)}\right) S=0 .
\end{array}\right.
$$

By calculating, we obtain the Jacobian matrix of $(F(\rho, J, S), G(\rho, J, S))$ at point $\left(0, J^{*}, S^{*}\right)$ is

$$
\left(\begin{array}{cc}
1-r_{1}+r_{1} c+\frac{\xi \beta S^{*}}{\left.\left(1+\beta U^{*}+S^{*}\right)\right)^{2}} & -\frac{\left.\xi(1+\beta)^{\prime \prime}\right)}{\left.\left(1+\beta U^{\prime \prime}+S^{*}\right)\right)^{2}} \\
-r_{1} c & 1-r_{2}
\end{array}\right) .
$$

It is easy to see that $F, G \in C^{k}\left(R_{+} \times R_{+}^{2}, R^{2}\right)$ for any integer $1 \leq k<\infty$, and $F\left(0, J^{\prime \prime}, S^{*}\right)=0$ and $G\left(0, J^{\prime \prime}, S^{*}\right)=0$. Combining $\left(\mathrm{H}_{4}\right)$, by using the existence theorem of implicit function, there exists an open subset $I_{1} \times I_{2}$ including $\left(0, J^{*}, S^{*}\right)$ in $R_{+} \times R_{+}^{2}$ such that for every $\rho$ in $I_{1}$, there exists a unique $\left(J_{\rho}, S_{\rho}\right)$ in $I_{2}$ such that $F\left(\rho, J_{\rho}, S_{\rho}\right)=0, G\left(\rho, J_{\rho}, S_{\rho}\right)=0$ and $\left(J_{0}, S_{0}\right)=\left(J^{*}, S^{*}\right)$. Furthermore, $J_{\rho}$ and $S_{\rho}$ are continuously differentiable with respect to $\rho$. This shows that there exists a positive constant $\delta_{1}>0$ with $\delta_{1}<1$ such that when $\rho \in\left[0, \delta_{1}\right)$, model (7) has an equilibrium $E_{\rho}^{*}\left(U_{\rho}^{*}, S_{\rho}^{*}\right)$ and

$$
\lim _{\rho \rightarrow 0} E_{\rho}^{*}\left(J_{\rho}^{*}, S_{\rho}^{*}\right)=E^{*}\left(J^{*}, S^{*}\right)
$$


The Jacobian matrix of model (7) is

$$
\Delta_{\rho}=\left(\begin{array}{cc}
r_{1}-r_{1} c-\frac{\xi \beta S(n)}{(1+\beta(J(n)+S(n)+\rho))^{2}} & \frac{\xi(1+\beta J(n)+\beta \rho)}{(1+\beta(J(n)+S(n)+\rho))^{2}} \\
r_{1} c+\frac{r_{2} \alpha \rho S(n)}{U(n)+S(n)+\rho)^{2}} & r_{2}-\frac{r_{2} \alpha \rho(J(n)+\rho)}{U(n)+S(n)+\rho)^{2}}
\end{array}\right) .
$$

Since

$$
\frac{\xi \beta S(n)}{(1+\beta(J(n)+S(n)+\rho))^{2}}<\frac{\xi}{4}, \quad \frac{J(n)+\rho}{(J(n)+S(n)+\rho)^{2}}<\frac{1}{\rho},
$$

it follows from $\left(\mathrm{H}_{1}\right)$ to $\left(\mathrm{H}_{3}\right)$ that for any $\rho \in\left(0, \delta_{1}\right)$, matrix $\Delta_{\rho}$ is positive, that is, all elements of $\Delta_{\rho}$ are positive.

Choose constant $\varepsilon>0$ such that $B^{*}>1+\varepsilon$. Let $K=1+\varepsilon / 2$. Further choose positive constants $\delta_{1}^{*}, \delta_{2}$ and $\delta_{1}^{*} \leq \delta_{1}$ such that $K \beta \rho<\varepsilon / 2$ and

$$
\delta_{2} \beta\left(K+\frac{r_{1} c}{1-r_{2}(1-\alpha)}\right)<\frac{\varepsilon}{4}
$$

for all $\rho \in\left(0, \delta_{1}^{*}\right)$. Therefore, for any positive solution $\left(J_{\rho}(n), S_{\rho}(n)\right)$ of model $(7)$, if $0<$ $J_{\rho}(0)<\delta_{2}$

$$
S_{\rho}(0)=\frac{J_{\rho}(0) r_{1} c}{\left(1-r_{2}(1-\alpha)\right) K}
$$

and $\rho \in\left(0, \delta_{1}^{*}\right)$, then we have

$$
\begin{aligned}
J_{\rho}(1)-J_{\rho}(0) & =\frac{\xi S_{\rho}(0)}{1+\beta\left(J_{\rho}(0)+S_{\rho}(0)+\rho\right)}+r_{1} J_{\rho}(0)-r_{1} c J_{\rho}(0)-J_{\rho}(0) \\
& =J_{\rho}(0)\left[\frac{B^{*}\left(1-r_{1}-r_{1} c\right)}{K+J_{\rho}(0) \beta\left(K+\frac{r_{1} c}{1-r_{2}(1-\alpha)}\right)+K \beta \rho}-\left(1-r_{1}+r_{1} c\right)\right] \\
& >J_{\rho}(0)\left[\frac{B^{*}\left(1-r_{1}-r_{1} c\right)}{K+\varepsilon / 2}-\left(1-r_{1}+r_{1} c\right)\right] \\
& =J_{\rho}(0)\left[\frac{B^{*}\left(1-r_{1}-r_{1} c\right)}{1+\varepsilon}-\left(1-r_{1}+r_{1} c\right)\right] \\
& =J_{\rho}(0)\left(1-r_{1}+r_{1} c\right)\left(\frac{B^{*}}{1+\varepsilon}-1\right) \\
& >0
\end{aligned}
$$

and

$$
\begin{aligned}
S_{\rho}(1)-S_{\rho}(0) & =r_{1} c J_{\rho}(0)+r_{2}\left(1-\frac{\alpha \rho}{J_{\rho}(0)+S_{\rho}(0)+\rho}\right) S_{\rho}(0)-S_{\rho}(0) \\
& >r_{1} c J_{\rho}(0)-\left(1-r_{2}(1-\alpha)\right) \frac{J_{\rho}(0) r_{1} c}{\left(1-r_{2}(1-\alpha)\right) K} \\
& =J_{\rho}(0) r_{1} c\left(1-\frac{1}{K}\right) \\
& >0 .
\end{aligned}
$$


Owing to the positivity of matrix $\Delta_{\rho}$, we obtain

$$
J_{\rho}(n+1)>J_{\rho}(n), \quad S_{\rho}(n+1)>S_{\rho}(n) \quad \text { for all } n \in Z \text {. }
$$

This shows that solution $\left(J_{\rho}(n), S_{\rho}(n)\right)$ of model (7) is increasing with respect to $n \in Z$. Therefore, we finally have

$$
\lim _{n \rightarrow \infty}\left(J_{\rho}(n), S_{\rho}(n)\right)=E_{\rho}^{*}\left(J_{\rho}^{*}, S_{\rho}^{*}\right)
$$

Further, choose constants $\varepsilon>0$ and $\Delta>0$ large enough such that $A^{*}>1+\varepsilon$ and

$$
\frac{A^{*} K}{1+\Delta \beta\left(1+\frac{r_{1} c K}{1-r_{2}}\right)}<1-\varepsilon,
$$

where $A^{*}$ is given in (5). For any positive solution $\left(J_{\rho}(n), S_{\rho}(n)\right)$ of model $(7)$, when $J_{\rho}(0)>\Delta$, $S_{\rho}(0)=J_{\rho}(0) r_{1} c K /\left(1-r_{2}\right)$ and $\rho \in\left(0, \delta_{1}^{*}\right)$, we have

$$
\begin{aligned}
J_{\rho}(1)-J_{\rho}(0) & =\frac{\xi S_{\rho}(0)}{1+\beta\left(J_{\rho}(0)+S_{\rho}(0)+\rho\right)}+r_{1} J_{\rho}(0)-r_{1} c J_{\rho}(0)-J_{\rho}(0) \\
& =\frac{\frac{J_{\rho}(0) \xi r_{1} c K}{\left(1-r_{2}\right)}}{1+\beta\left(J_{\rho}(0)+\frac{J_{\rho}(0) r_{1} c K}{\left(1-r_{2}\right)}+\rho\right)}+r_{1} J_{\rho}(0)-r_{1} c J_{\rho}(0)-J_{\rho}(0) \\
& \leq J_{\rho}(0)\left[\frac{A^{*} K\left(1-r_{1}+r_{1} c\right)}{1+\Delta \beta\left(1+\frac{r_{1} c K}{1-r_{2}}\right)}-\left(1-r_{1}+r_{1} c\right)\right] \\
& <-\varepsilon J_{\rho}(0)\left(1-r_{1}+r_{1} c\right) \\
& <0
\end{aligned}
$$

and

$$
\begin{aligned}
S_{\rho}(1)-S_{\rho}(0) & =r_{1} c J_{\rho}(0)+r_{2}\left(1-\frac{\alpha \rho}{J_{\rho}(0)+S_{\rho}(0)+\rho}\right) S_{\rho}(0)-S_{\rho}(0) \\
& <r_{1} c J_{\rho}(0)-\left(1-r_{2}\right) \frac{J_{\rho}(0) r_{1} c K}{\left(1-r_{2}\right)} \\
& =J_{\rho}(0) r_{1} c(1-K) \\
& <0
\end{aligned}
$$

Similarly, owing to the positivity of matrix $\Delta_{\rho}$, we obtain $J_{\rho}(n+1)<J_{\rho}(n)$ and $S_{\rho}(n+1)<$ $S_{\rho}(n)$ for all $n \in Z$. This shows that solution $\left(J_{\rho}(n), S_{\rho}(n)\right)$ of model (7) is decreasing with respect to $n \in Z$. Therefore, we finally also have $\lim _{n \rightarrow \infty}\left(J_{\rho}(n), S_{\rho}(n)\right)=E_{\rho}^{*}\left(J_{\rho}^{*}, S_{\rho}^{*}\right)$.

Let $\left(J_{\rho}(n), S_{\rho}(n)\right)$ be any positive solution of model (7). Choose two solutions $\left(\bar{J}_{\rho}(n), \bar{S}_{\rho}(n)\right)$ and $\left.\hat{J}_{\rho}(n), \hat{S}_{\rho}(n)\right)$ of model (7) satisfying

$$
0<\bar{J}_{\rho}(0)<\delta_{2}, \quad \bar{S}_{\rho}(0)=\frac{\bar{J}_{\rho}(0) r_{1} c}{\left(1-r_{2}(1-\alpha)\right) K}
$$

and

$$
\hat{J}_{\rho}(0)>\Delta, \quad \hat{S}_{\rho}(0)=\frac{\hat{J}_{\rho}(0) r_{1} c K}{1-r_{2}},
$$


respectively, such that $\bar{J}_{\rho}(0)<J_{\rho}(0)<\hat{J}_{\rho}(0)$ and $\bar{S}_{\rho}(0)<S_{\rho}(0)<\hat{S}_{\rho}(0)$, then when $\rho \in\left(0, \delta_{1}^{*}\right)$, owing to the positivity of matrix $\Delta_{\rho}$, we obtain $\bar{J}_{\rho}(n)<J_{\rho}(n)<\hat{J}_{\rho}(n)$ and $\bar{S}_{\rho}(n)<S_{\rho}(n)<$ $\hat{S}_{\rho}(n)$ for all $n \in Z$. Therefore, we finally have $\lim _{n \rightarrow \infty}\left(U_{\rho}(n), S_{\rho}(n)\right)=E_{\rho}^{*}\left(U_{\rho}^{*}, S_{\rho}^{*}\right)$. This completes the proof of Lemma 2.2.

Directly from Lemma 2.2, we have the following result.

Corollary 2.1 Assume that $\left(\mathrm{H}_{1}\right)$ to $\left(\mathrm{H}_{4}\right)$ hold and $A^{*}>1$. Then $E^{*}\left(U^{*}, S^{*}\right)$ is a globally asymptotically stable equilibrium of model (3).

In the following, we introduce the comparison principle of difference equations.

Lemma 2.3 (see [21]) Suppose that functions $f, g: Z_{+} \times[0, \infty) \rightarrow[0, \infty)$ satisfy $f(n, x) \leq$ $g(n, x)$ (or $f(n, x) \geq g(n, x))$ for $n \in Z_{+}$and $x \in[0, \infty)$ and $g(n, x)$ is nondecreasing with respect to $x \geq 0$. If sequences $\{x(n)\}$ and $\{u(n)\}$ are the nonnegative solutions of the following difference equations

$$
x(n+1)=f(n, x(n)), \quad u(n+1)=g(n, u(n)), \quad n=0,1,2, \ldots,
$$

respectively, and $x(0) \leq u(0)($ or $x(0) \geq u(0))$, then we have

$$
x(n) \leq u(n) \quad(\text { or } x(n) \geq u(n)) \quad \text { for all } n \geq 0 .
$$

For any $x=\left(x_{1}, x_{2}, \ldots, x_{n}\right) \in R^{n}$ we define $|x|=\max \left\{\left|x_{1}\right|,\left|x_{2}\right|, \ldots,\left|x_{n}\right|\right\}$. For any constant $B>0$, let $S_{B}=\left\{x \in R^{n}:|x|<B\right\}$. Suppose that $F(x): R^{n} \rightarrow R^{n}$ be a continuous map, we consider the following difference equation:

$$
x(n+1)=F(x(n)), \quad n \in Z
$$

On the existence of equilibrium of model (9), we have the following result.

Lemma 2.4 Assume that model (9) is ultimately bounded with respect to $B$, that is, there is a constant $B>0$ such that for any solution $x(n)=\left(x_{1}(n), x_{2}(n), \ldots, x_{n}(n)\right)$ of model $(9)$

$$
\limsup _{n \rightarrow \infty}\left|x_{i}(n)\right| \leq B, \quad i=1,2, \ldots, n
$$

Then model (9) has at least an equilibrium.

Proof Let $x\left(n, x_{0}\right)$ be the solution of model (9) with initial value $x(0)=x_{0} \in R^{n}$. From the continuity of $F(x)$, we can obtain that $x\left(n, x_{0}\right)$ is continuous with respect to $x_{0} \in R^{n}$. Firstly, we prove the following claims.

Claim 2.1 For any constant $A>B$, there is a constant $\lambda>A$ such that $\left|x\left(n, x_{0}\right)\right|<\lambda$ for all $n \in Z$ and $x_{0} \in \bar{S}_{A}$, where $\bar{S}_{A}$ is the closure of set $S_{A}$.

Otherwise, there are two sequences $\left\{x_{k}\right\} \subset \bar{S}_{A}$ and $\left\{n_{k}\right\}$ such that

$$
\lim _{k \rightarrow \infty}\left|x\left(n_{k}, x_{k}\right)\right|=\infty
$$


Then there is a $k_{1}>0$ such that $\left|x\left(n_{k}, x_{k}\right)\right|>A$ for all $k \geq k_{1}$. Since $\left|x\left(0, x_{k}\right)\right|=\left|x_{k}\right|<A$ for all $k \in Z$, there is a $s_{k} \in\left[0, n_{k}\right)$ such that $\left|x\left(s_{k}, x_{k}\right)\right| \leq A$ and $\left|x\left(n, x_{k}\right)\right|>A$ for all $n \in\left(s_{k}, n_{k}\right]$ and $k \geq k_{1}$. Let $\psi_{k}=x\left(s_{k}, x_{k}\right)$, then $\psi_{k} \in \bar{S}_{A}$ for all $k \geq k_{1}$. Without loss of generality, we can assume that there is a $\psi_{0} \in \bar{S}_{A}$ such that $\lim _{k \rightarrow \infty} \psi_{k}=\psi_{0}$.

We consider solution $x\left(n, \psi_{0}\right)$ of model (9), by the ultimate boundedness of model (9), there is an $N_{0}>0$ such that $\left|x\left(n, \psi_{0}\right)\right|<B$ for all $n \geq N_{0}$. From the continuity of solution $x\left(n, x_{0}\right)$ with respect to $x_{0} \in R^{n}$, there is an integer $N_{1}>0$ such that $\left|x\left(N_{0}, \psi_{k}\right)\right|<B$ for all $k \geq N_{1}$. Choose a constant $M_{1}>\max \left\{A,\left|x\left(n, \psi_{0}\right)\right|: n \in\left[0, N_{0}\right]\right\}$, then there is an integer $N_{2}>N_{1}$ such that $\left|x\left(n, \psi_{k}\right)\right|<M_{1}$ for all $k \geq N_{2}$ and $n \in\left[0, N_{0}\right]$. Since $x\left(N_{0}, \psi_{k}\right)=$ $x\left(N_{0}, x\left(s_{k}, x_{k}\right)\right)=x\left(N_{0}+s_{k}, x_{k}\right)$, we obtain $\left|x\left(N_{0}+s_{k}, x_{k}\right)\right|<B<A$ for all $k \geq N_{1}$. On the other hand, from $\lim _{k \rightarrow \infty}\left|x\left(n_{k}, x_{k}\right)\right|=\infty$, there is an $N_{3}>N_{2}$ such that $\left|x\left(n_{k}, x_{k}\right)\right|>M_{1}>A$ for all $k \geq N_{3}$. Thus, we finally have $s_{k}<N_{0}+s_{k}<n_{k}$. Consequently, $\left|x\left(N_{0}+s_{k}, x_{k}\right)\right|>A$, which leads to a contradiction with $\left|x\left(N_{0}+s_{k}, x_{k}\right)\right|<A$. Therefore, Claim 2.1 is true.

By Claim 2.1, we can choose three constants $B_{0}<B_{1}<B_{2}$ such that $\left|x\left(n, x_{0}\right)\right|<B_{0}$ for all $x_{0} \in \bar{S}_{B}$ and $n \in Z,\left|x\left(n, x_{0}\right)\right|<B_{1}$ for all $x_{0} \in \bar{S}_{B_{0}}$ and $n \in Z,\left|x\left(n, x_{0}\right)\right|<B_{2}$ for all $x_{0} \in \bar{S}_{B_{1}}$ and $n \in Z$. Let $S_{0}=\bar{S}_{B_{0}}, S_{1}=S_{B_{1}}$ and $S_{2}=\bar{S}_{B_{2}}$. Then $S_{0}, S_{1}$ and $S_{2}$ are convex subsets of $R^{n}$ with $S_{0}$ and $S_{2}$ compact and $S_{1}$ open in $S_{2}$.

Next, we prove the following claim.

Claim 2.2 There is an integer $T_{0}>0$ such that $\left|x\left(n, x_{0}\right)\right|<B_{0}$ for all $x_{0} \in S_{2}$ and $n \geq T_{0}$.

Otherwise, there exist two sequences $\left\{x_{k}\right\} \subset S_{2}$ and $\left\{t_{k}\right\}$ satisfying $t_{k} \rightarrow \infty$ as $k \rightarrow \infty$ such that $\left|x\left(t_{k}, x_{k}\right)\right| \geq B_{0}$ for all $k \in Z$. Without loss of generality, we can assume that there is a $x_{0} \in S_{2}$ such that $\lim _{k \rightarrow \infty} x_{k}=x_{0}$.

By the ultimate boundedness of model (9), there exists an integer $q \in Z$ such that $\left|x\left(n, x_{0}\right)\right|<B$ for all $n>q$. From the continuity of solution $x\left(n, x_{0}\right)$ of model (9) with respect to $x_{0} \in R^{n}$, there exists an integer $J_{0} \in Z$ such that $\left|x\left(n, x_{k}\right)\right|<B$ for $n \in[q, 2 q]$ and $k>J_{0}$. This implies that $\left|x\left(2 q, x_{k}\right)\right|<B$. Let $\psi_{k}=x\left(2 q, x_{k}\right)$, then we have $\left|x\left(n, \psi_{k}\right)\right|<B_{0}$ for all $n \in Z$. Since $x\left(n, \psi_{k}\right)=x\left(n+2 q, x_{k}\right)$ for all $n \geq 0$, we have $\left|x\left(n, x_{k}\right)\right|<B_{0}$ for all $n \geq 2 q$ and $j>J_{0}$ which leads to a contradiction with $\left|x\left(t_{k}, x_{k}\right)\right| \geq B_{0}$ if $t_{k} \geq 2 q$. Therefore, Claim 2.2 is true.

Define map $P: R^{n} \rightarrow R^{n}$ as follows:

$$
P\left(x_{0}\right)=x\left(1, x_{0}\right)=F\left(x_{0}\right), \quad x_{0} \in R^{n} .
$$

Then we have $P^{j}\left(S_{1}\right) \subset S_{2}$ for all $j \in N$. Choose an integer $m \in N$ such that $m>T_{0}$, where $T_{0}$ is given in Claim 2.2. From Claim 2.2, we can obtain that $P^{j}\left(S_{2}\right) \subset S_{0}$ for all $j \geq m$.

From the above discussion, we see that all conditions of the Horn fixed-point theorem (see [22]) are satisfied. Therefore, map $P$ has at least a fixed point $x_{0} \in R^{n}$. Consequently, model (9) has at least an equilibrium $x_{0} \in R^{n}$. This completes the proof of Lemma 2.4.

In order to discuss the local stability of endemic equilibrium of model (1), we also need the following result (see [22, Lemma 2.2]).

Lemma 2.5 Let equation $x^{3}+b x^{2}+c x+d=0$, where $b, c, d \in R$. Let further $A=b^{2}-3 c$, $B=b c-9 d, C=c^{2}-3 b d$ and $\Delta=B^{2}-4 A C$. Then we have

(1) The equation has three different real roots if and only if $\Delta \leq 0$; 
(2) The equation has one real root and a pair of conjugate complex roots if and only if $\Delta>0$. Further, the conjugate complex roots are

$$
w=\frac{-2 b+Y_{1}^{\frac{1}{3}}+Y_{2}^{\frac{1}{3}}}{6} \pm i \frac{\sqrt{3}\left(Y_{1}^{\frac{1}{3}}-Y_{2}^{\frac{1}{3}}\right)}{6}
$$

where

$$
Y_{1,2}=b A+\frac{-B \pm \sqrt{B^{2}-4 A C}}{2} .
$$

\section{Permanence and extinction}

Firstly, on the ultimate boundedness of all positive solutions of model (1), we have the following result.

Theorem 3.1 Assume that $\left(\mathrm{H}_{1}\right)$ and $\left(\mathrm{H}_{2}\right)$ hold. Then for any positive solution $(J(n), S(n)$, $I(n))$ of model (1),

$$
\limsup _{n \rightarrow \infty} N(n) \leq \frac{\xi+R-1}{(1-R) \beta}(R+\xi)^{2}=: M .
$$

Proof Since $0<R<1$ and $\xi+R>1$, there exists a constant $N_{0}>0$ such that $\xi /\left(1+\beta N_{0}\right)+$ $R=1$. Obviously,

$$
N_{0}=\frac{\xi+R-1}{(1-R) \beta} .
$$

We consider the following three cases.

Case I. There exists an integer $n_{0} \in Z$ such that

$$
N(n) \leq \frac{\xi+R-1}{(1-R) \beta}(R+\xi) \quad \text { for all } n \geq n_{0} .
$$

Then the conclusion of Theorem 3.1 is obviously true in this case.

Case II. There exists $n_{0} \in Z$ such that

$$
N(n)>\frac{\xi+R-1}{(1-R) \beta}(R+\xi) \quad \text { for all } n \geq n_{0}
$$

Then, for $n>n_{0}$, we have

$$
\begin{aligned}
N(n+1) & =\frac{\xi S(n)}{1+\beta N(n)}+r_{1} J(n)+r_{2} S(n)+r_{3} I(n) \\
& \leq \frac{\xi N(n)}{1+\beta N(n)}+R N(n) \\
& =N(n)\left[\frac{\xi}{1+\beta N(n)}+R\right] \\
& <N(n)\left[\frac{\xi}{1+\beta N_{0}(\xi+R)}+R\right] .
\end{aligned}
$$

It follows from $\left(\mathrm{H}_{2}\right)$ and $(11)$ that

$$
\frac{\xi}{1+\beta N_{0}(\xi+R)}+R<1,
$$


which implies lim $\sup _{n \rightarrow \infty} N(n)=0$. This leads to a contradiction with (12). Therefore, the statement of Case II is false.

Case III. $N(n)$ oscillates about $(\xi+R-1)(R+\xi) /(1-R) \beta$.

Obviously, we only need to consider thus $N(n)$, which satisfies

$$
N(n) \geq \frac{\xi+R-1}{\beta(1-R)}(R+\xi) .
$$

Consider set $\{N(l+1), N(l+2), \ldots, N(l+L)\}$ with $l \geq 0$ and $L<\infty$ satisfying

$$
N(l+i+1) \geq \frac{\xi+R-1}{(1-R) \beta}(R+\xi) \quad \text { for all } 0 \leq i \leq L-1 .
$$

From (13), we have

$$
\begin{aligned}
N(l+i+1) & \leq N(l+i)\left[\frac{\xi}{1+\beta N(l+i)}+R\right] \\
& <N(l+i)\left[\frac{\xi}{1+\beta N_{0}(\xi+R)}+R\right] \\
& <N(l+i),
\end{aligned}
$$

which implies that $\max \{N(l+1), N(l+2), \ldots, N(l+L)\}=N(l+1)$. Owing to $N(l)<N_{0}(\xi+R)$, we hence have

$$
N(l+1) \leq N(l)\left[\frac{\xi}{1+\beta N(l)}+R\right] \leq N(l)(\xi+R) \leq N_{0}(\xi+R)^{2},
$$

which implies that (10) hold. This completes the proof of Theorem 3.1.

Next, on the permanence of all positive solutions of model (1), we have the following result.

Theorem 3.2 Assume that $\left(\mathrm{H}_{1}\right)$ to $\left(\mathrm{H}_{4}\right)$ hold, $B^{*}>1$ and $R_{0}>1$. Then the disease in model (1) is permanent, where

$$
R_{0}=\frac{r_{2} \alpha S^{*}}{M}+r_{3}(1-\sigma)
$$

$B^{*}, S^{*}$ and $M$ are given in (6), (8) and (10), respectively.

Proof Let $(J(n), S(n), I(n))$ be any positive solution of model (1), we need only prove that there exists constant $m_{3}>0$ such that

$$
\liminf _{n \rightarrow \infty} I(n) \geq m_{3}
$$

Firstly, from Theorem 3.1, there is an integer $n_{0}>0$ such that

$$
N(n)=J(n)+S(n)+I(n) \leq M \quad \text { for all } n \geq n_{0} .
$$


In view of $R_{0}>1$, there exists a small enough positive constant $\epsilon_{0}>0$ such that

$$
R_{0}^{\epsilon_{0}}=\frac{r_{2} \alpha\left(S^{*}-\epsilon_{0}\right)}{M}+r_{3}(1-\sigma)>1
$$

From Lemma 2.2, for above $\epsilon_{0}>0$ there exists a constant $\delta=\delta\left(\epsilon_{0}\right)>0$ such that when $0<\rho<\delta$

$$
\left|J_{\rho}^{*}-J^{*}\right|<\frac{\epsilon_{0}}{2}, \quad\left|S_{\rho}^{*}-S^{*}\right|<\frac{\epsilon_{0}}{2},
$$

where $E^{*}\left(J^{*}, S^{*}\right)$ and $E_{\rho}^{*}\left(J_{\rho}^{*}, S_{\rho}^{*}\right)$ are the positive equilibria of model (3) and model (7), respectively. Set $\alpha_{0}=\delta / 2$. We consider the following three cases.

Case 1. There exists $n_{1} \geq n_{0}$ such that $I(n) \geq \alpha_{0}$ for all $n \geq n_{1}$.

In this case, we only need choose $m_{3}=\alpha_{0}$, then (14) hold.

Case 2. There exists $n_{1} \geq n_{0}$ such that $I(n)<\alpha_{0}$ for all $n \geq n_{1}$.

In this case, we can obtain for any $n \geq n_{1}$

$$
\begin{aligned}
& J(n+1) \geq \frac{\xi S(n)}{1+\beta\left(J(n)+S(n)+\alpha_{0}\right)}+r_{1} J(n)-r_{1} c J(n), \\
& S(n+1) \geq r_{1} c J(n)+r_{2}\left(1-\frac{\alpha \alpha_{0}}{J(n)+S(n)+\alpha_{0}}\right) S(n) .
\end{aligned}
$$

Let $\left(J_{\alpha_{0}}(n), S_{\alpha_{0}}(n)\right)$ be the positive solution of model (7) with $\rho=\alpha_{0}$ and initial value $\left(J_{\alpha_{0}}\left(n_{1}\right), S_{\alpha_{0}}\left(n_{1}\right)\right)=\left(J\left(n_{1}\right), S\left(n_{1}\right)\right)$. From Lemma 2.3, we have

$$
J(n) \geq J_{\alpha_{0}}(n), \quad S(n) \geq S_{\alpha_{0}}(n)
$$

for all $n \geq n_{1}$. Further, from Lemma 2.2, we obtain that equilibrium $E_{\alpha_{0}}^{*}\left(J_{\alpha_{0}}^{*}, S_{\alpha_{0}}^{*}\right)$ of model (7) with $\rho=\alpha_{0}$ is globally uniformly asymptotically stable. Hence, for above $\epsilon_{0}>0$ there exits $n_{2}>n_{1}$ such that

$$
\left|J_{\alpha_{0}}(n)-J_{\alpha_{0}}^{*}\right|<\frac{\epsilon_{0}}{2}, \quad\left|S_{\alpha_{0}}(n)-S_{\alpha_{0}}^{*}\right|<\frac{\epsilon_{0}}{2}
$$

for all $n \geq n_{2}$. Therefore, from (15)-(17), for any $n \geq n_{2}$, we can obtain

$$
\begin{aligned}
I(n+1) & =r_{2} \frac{\alpha I(n)}{J(n)+S(n)+I(n)} S(n)+r_{3}(1-\sigma) I(n) \\
& \geq r_{2} \frac{\alpha I(n)}{M} S(n)+r_{3}(1-\sigma) I(n) \\
& =I(n)\left(\frac{r_{2} \alpha S(n)}{M}+r_{3}(1-\sigma)\right) \\
& \geq I(n)\left(\frac{r_{2} \alpha S_{\alpha_{0}}(n)}{M}+r_{3}(1-\sigma)\right) \\
& >I(n)\left(\frac{r_{2} \alpha\left(S_{\alpha_{0}}^{*}-\epsilon_{0} / 2\right)}{M}+r_{3}(1-\sigma)\right) \\
& >I(n)\left(\frac{r_{2} \alpha\left(S^{*}-\epsilon_{0}\right)}{M}+r_{3}(1-\sigma)\right) .
\end{aligned}
$$


From this, we further have $\lim _{n \rightarrow \infty} I(n)=\infty$, which leads to a contradiction with $I(n)<\alpha_{0}$ for all $n \geq n_{1}$. Therefore, the statement of Case 2 is false.

Case 3. I $(n)$ oscillates about $\alpha_{0}$.

Obviously, there exists integer sequences $\left\{\tau_{k}\right\}_{k=1}^{\infty}$ and $\left\{t_{k}\right\}_{k=1}^{\infty}$ with $n_{0} \leq \tau_{1}<t_{1}<\cdots \tau_{k}<$ $t_{k}<\cdots$ and $\lim _{k \rightarrow \infty} \tau_{k}=\infty$ such that

$$
\begin{aligned}
& I(n)<\alpha_{0} \quad \text { for all } n \in\left[\tau_{k}, t_{k}\right], \\
& I(n) \geq \alpha_{0} \quad \text { for all } n \notin\left[\tau_{k}, t_{k}\right] .
\end{aligned}
$$

When $n \in\left[\tau_{k}, t_{k}\right]$ for any $k \in Z$, we have

$$
\begin{aligned}
& J(n+1) \geq \frac{\xi S(n)}{1+\beta\left(J(n)+S(n)+\alpha_{0}\right)}+r_{1} J(n)-r_{1} c J(n), \\
& S(n+1) \geq r_{1} c J(n)+r_{2}\left(1-\frac{\alpha \alpha_{0}}{J(n)+S(n)+\alpha_{0}}\right) S(n) .
\end{aligned}
$$

From Lemma 2.3, we have

$$
J(n) \geq J_{\alpha_{0}}(n), \quad S(n) \geq S_{\alpha_{0}}(n)
$$

for all $n \in\left[\tau_{k}, t_{k}\right]$, where $\left(J_{\alpha_{0}}(n), S_{\alpha_{0}}(n)\right)$ is the positive solution of model (7) with $\rho=\alpha_{0}$ and initial value $\left(J_{\alpha_{0}}\left(\tau_{k}\right), S_{\alpha_{0}}\left(\tau_{k}\right)\right)=\left(J\left(\tau_{k}\right), S\left(\tau_{k}\right)\right)$. Since equilibrium $E_{\alpha_{0}}^{*}\left(J_{\alpha_{0}}^{*}, S_{\alpha_{0}}^{*}\right)$ of model (7) with $\rho=\alpha_{0}$ is globally uniformly asymptotically stable, for above $\epsilon_{0}>0$ there exits an integer $\bar{n}>0$ which is independent of any $\tau_{k}$ and $\left(J\left(\tau_{k}\right), S\left(\tau_{k}\right)\right)$ such that

$$
\left|J_{\alpha_{0}}(n)-J_{\alpha_{0}}^{*}\right|<\frac{\epsilon_{0}}{2}, \quad\left|S_{\alpha_{0}}(n)-S_{\alpha_{0}}^{*}\right|<\frac{\epsilon_{0}}{2}
$$

for all $n \geq \tau_{k}+\bar{n}$. We claim that there exists an integer $N_{0} \in Z$ depending on only $\epsilon_{0}$ such that

$$
t_{k}-\tau_{k}-\bar{n}+1 \leq N_{0} .
$$

Otherwise, for any large enough $G>0$, there exists an integer $K>0$ such that

$$
t_{K}-\tau_{K}-\bar{n}+1>G
$$

We choose

$$
G=-\frac{(\bar{n}+1) \ln \left(r_{3}(1-\sigma)\right)}{\ln R_{0}^{\epsilon}}+1
$$

From (15) and (18)-(20), we can obtain that when $n \in\left[\tau_{k}+\bar{n}, t_{k}\right]$

$$
\begin{aligned}
I(n+1) & =\frac{r_{2} \alpha I(n)}{J(n)+S(n)+I(n)} S(n)+r_{3}(1-\sigma) I(n) \\
& \geq I(n)\left(\frac{r_{2} \alpha S(n)}{M}+r_{3}(1-\sigma)\right)
\end{aligned}
$$




$$
\begin{aligned}
& \geq I(n)\left(\frac{r_{2} \alpha S_{\alpha_{0}}(n)}{M}+r_{3}(1-\sigma)\right) \\
& >I(n)\left(\frac{r_{2} \alpha\left(S_{\alpha_{0}}^{*}-\epsilon_{0} / 2\right)}{M}+r_{3}(1-\sigma)\right) \\
& >I(n)\left(\frac{r_{2} \alpha\left(S^{*}-\epsilon_{0}\right)}{M}+r_{3}(1-\sigma)\right) .
\end{aligned}
$$

Hence, we further obtain

$$
\begin{aligned}
\alpha_{0} & \geq I\left(t_{k}\right) \\
& >I\left(\tau_{k}+\bar{n}\right)\left(R_{0}^{\varepsilon}\right)^{t_{k}-\tau_{k}-\bar{n}} \\
& \geq I\left(\tau_{k}-1\right)\left[r_{0}(1-\sigma)\right]^{\bar{n}+1}\left(R_{0}^{\varepsilon}\right)^{t_{k}-\tau_{k}-\bar{n}} \\
& \geq \alpha_{0}\left[r_{0}(1-\sigma)\right]^{\bar{n}+1}\left[R_{0}^{\epsilon}\right]^{G-1} \\
& =\alpha_{0} .
\end{aligned}
$$

This contradiction implies that the above claim is true. Thus, for any $n \in\left[\tau_{k}, t_{k}\right]$, we have

$$
\begin{aligned}
I(n) & =\frac{r_{2} \alpha I(n-1)}{J(n-1)+S(n-1)+I(n-1)} S(n-1)+r_{3}(1-\sigma) I(n-1) \\
& \geq I(n-1) r_{3}(1-\sigma) \\
& \geq I\left(\tau_{k}-1\right)\left[r_{3}(1-\sigma)\right]^{n-\tau_{k}+1} \\
& \geq \alpha_{0}\left[r_{3}(1-\sigma)\right]^{N_{0}+\bar{n}} .
\end{aligned}
$$

Choose $m_{3}=\alpha_{0}\left[r_{3}(1-\sigma)\right]^{N_{0}+\bar{n}}$, then (14) is true. This completes the proof of Theorem 3.2.

Remark 3.1 From the expression of constant $M$ given in Theorem 3.1, we see that $M$ is a quite big positive constant. This shows that condition $R_{0}>1$ in Theorem 3.2 is quite strong. Therefore, an important and interesting open problem is to establish a more precise result on the permanence of the disease for model (1).

Further, on the extinction of the disease in model (1) we have the following result.

Theorem 3.3 Assume that $\left(\mathrm{H}_{1}\right)$ to $\left(\mathrm{H}_{4}\right)$ hold, $B^{*}>1$ and $R_{1}<1$, where $R_{1}=r_{2} \alpha+r_{3}(1-\sigma)$ and $B^{\prime \prime}$ is given in (8). Then disease-free equilibrium $\left(J^{\prime \prime}, S^{\prime \prime}, 0\right)$ of model $(1)$ is globally stable. This shows that the disease in model (1) is extinct.

The proof of Theorem 3.3 simple. In fact, for any positive solution $(J(n), S(n), I(n))$ of model (1), directly from model (1) we have

$$
\begin{aligned}
I(n+1) & =r_{2} \alpha \frac{I(n)}{N(n)} S(n)+r_{3}(1-\sigma) I(n) \\
& \leq r_{2} \alpha I(n)+r_{3}(1-\sigma) I(n) \\
& =R_{1} I(n) .
\end{aligned}
$$


From this, we immediately obtain $\lim _{n \rightarrow \infty} I(n)=0$. A similar argument as in the proof of Lemma 2.2 we can obtain that $J(n) \rightarrow J^{*}$ and $S(n) \rightarrow S^{*}$ as $n \rightarrow \infty$. This shows that disease-free equilibrium $\left(J^{*}, S^{*}, 0\right)$ of model (1) is globally stable. Consequently, the disease in model (1) is also extinct.

Remark 3.2 Comparing $R_{0}$ with $R_{1}$, since $M \geq S^{\prime \prime}$, we have $R_{0} \leq R_{1}$. Therefore, combining the results given in Theorem 3.2 and Theorem 3.3, we can give the following important and interesting open problem. That is, whether there exists a threshold value $R^{*} \in\left[R_{0}, R_{1}\right]$ such that when $R^{*}<1$ then the disease in model (1) is extinct and when $R^{*}>1$ then the disease in model (1) is permanent.

\section{Existence and stability of endemic equilibrium}

Firstly, directly applying Lemma 2.4, we have the following result on the existence of the endemic equilibrium of model (1).

Theorem 4.1 Assume that all conditions of Theorem 3.2 hold. Then model (1) has at least one endemic equilibrium $(\hat{J}, \hat{S}, \hat{I})$.

Proof Firstly, for the following linear difference equation:

$$
x(n+1)=\alpha x(n)+\beta, \quad n \in Z,
$$

where $\alpha$ and $\beta$ are constants, we have that if $0<\alpha<1$ and $\beta>0$ then any solution $x(n)$ of equation (21) satisfies $\lim _{n \rightarrow \infty} x(n)=\beta /(1-\alpha)$. Therefore, we can choose an integer $N_{0}>0$ such that $x(n) \geq \beta / 2(1-\alpha)$ for all $n \geq N_{0}$.

Let $(J(n), S(n), I(n))$ be any positive solution of model (1). From Theorem 3.2, there are constants $M_{1}>m_{1}>0$ which are independent of any positive solution of model (1) and there exists an integer $N_{1}>0$ such that

$$
m_{1} \leq I(n) \leq M_{1}, \quad N(n)=J(n)+S(n)+I(n) \leq M \quad \text { for all } n \geq N_{1},
$$

where $M$ is given in (10). From this, we further have for any $n \geq N_{1}$

$$
S(n+1) \geq r_{2}(1-\alpha) S(n)+r_{3} \sigma m_{1} .
$$

From the above discussion, we can obtain that there is an integer $N_{2}>N_{1}$ such that for $n \geq N_{2}$

$$
S(n) \geq \frac{r_{3} \sigma m_{1}}{2\left(1-r_{2}(1-\alpha)\right)}:=m_{2} .
$$

From this, we further have for any $n \geq N_{2}$

$$
J(n+1) \geq r_{1}(1-c) J(n)+\frac{\xi m_{2}}{1+\beta M} .
$$

Again from the above discussion, we obtain that there is an integer $N_{3}>N_{2}$ such that

$$
J(n) \geq \frac{\xi m_{2}}{2\left(1-r_{1}+r_{1} c\right)(1+\beta M)}:=m_{3}
$$


for all $n \geq N_{3}$. From (22)-(24), we see that model (1) is permanent. Therefore, by Lemma 2.4 we can obtain that model (1) has at least an endemic equilibrium. This completes the proof of this Theorem 4.1.

Remark 4.1 From Theorem 4.1, we obtain that if model (1) is permanent, then model (1) has at least an endemic equilibrium.

Further, let

$$
R_{0}^{*}=\frac{\xi\left(1-r_{3}+r_{3} \sigma\right)\left(1-r_{3}+r_{1} c\right)}{\left[\left(1-r_{3}\right) r_{2} \alpha-\left(r_{2}-r_{3}\right)\left(1-r_{3}+r_{3} \sigma\right)\right]\left(1-r_{1}+r_{1} c\right)}
$$

and

$$
R_{1}^{*}=\frac{r_{1} c\left[\left(1-r_{3}\right) r_{2} \alpha-\left(r_{2}-r_{3}\right)\left(1-r_{3}+r_{3} \sigma\right)\right]+r_{2}\left(1-r_{3}+r_{3} \sigma\right)\left(1-r_{3}+r_{1} c\right)}{\left(1-r_{3}+r_{3} \sigma\right)\left(1-r_{3}+r_{1} c\right)}
$$

We see that endemic equilibrium $(\hat{J}, \hat{S}, \hat{I})$ of model (1) satisfies the following algebra equations:

$$
\begin{aligned}
& J=\frac{\xi S}{1+\beta(J+S+I)}+r_{1} J-r_{1} c J, \\
& S=r_{1} c J+r_{2}\left(1-\frac{\alpha I}{J+S+I}\right) S+r_{3} \sigma I, \\
& I=r_{2} \alpha \frac{I S}{J+S+I}+r_{3}(1-\sigma) I .
\end{aligned}
$$

From the third equation of (25), it follows that

$$
\hat{S}=\frac{\left(1-r_{3}+r_{3} \sigma\right)}{r_{2} \alpha}(\hat{J}+\hat{S}+\hat{I}) .
$$

Further, from this and the first equation of (25) we have

$$
\hat{J}=\frac{\xi \hat{S}}{(1+\beta(\hat{J}+\hat{S}+\hat{I}))\left(1-r_{1}+r_{1} c\right)} .
$$

This together with (26), (27), the second and third equations of (25), we can get that

$$
\hat{I}=\frac{r_{1} c \hat{J}+r_{2} \hat{S}-\hat{S}}{1-r_{3}}
$$

Then, it follows that

$$
\hat{J}+\hat{S}+\hat{I}=\frac{R_{0}^{*}-1}{\beta} .
$$

Therefore, when $\hat{J}+\hat{S}+\hat{I}>0$ and $\hat{I}>0$, namely $R_{0}^{*}>1$ and $R_{1}^{*}>1$, we can obtain that algebra equation (25) has a unique positive solution which are given in (26)-(29). Therefore, we obtain a result on the existence of the endemic equilibrium for model (1). 
Theorem 4.2 If $R_{0}^{*}>1$ and $R_{1}^{*}>1$, then model (1) has only an endemic equilibrium $(\hat{J}, \hat{S}, \hat{I})$ and which are given in (26)-(29).

Remark 4.2 On the existence of endemic equilibrium of model (1), we note that the method of Theorem 4.1 is totally different to the method in Theorem 4.2. From the expressions of $R_{0}$ and $B^{*}$ in Theorem 3.2, $R_{0}^{*}$ and $R_{1}^{*}$ of Theorem 4.2, we cannot get the relationship between them. However, we note that Theorem 4.1 implies Theorem 4.2. So, we have the following interesting conjecture.

Conjecture 4.1 If all conditions of Theorem 3.2 holds, then model (1) has only an endemic equilibrium $(\hat{J}, \hat{S}, \hat{I})$ and which are given in (26)-(29).

Next, we consider the stability of endemic equilibrium of model (1). The Jacobian matrix of model (1) at equilibrium $(\hat{J}, \hat{S}, \hat{I})$ is given

$$
J(\hat{J}, \hat{S}, \hat{I})=\left(\begin{array}{lll}
a_{11} & a_{12} & a_{13} \\
a_{21} & a_{22} & a_{23} \\
a_{31} & a_{32} & a_{33}
\end{array}\right),
$$

where

$$
\begin{aligned}
& a_{11}=r_{1}-r_{1} c-\frac{\xi \beta \hat{S}}{(1+\beta \hat{N})^{2}}, \quad a_{12}=\frac{\xi(1+\beta(\hat{J}+\hat{I}))}{(1+\beta \hat{N})^{2}}, \quad a_{13}=-\frac{\xi \beta \hat{S}}{(1+\beta \hat{N})^{2}}, \\
& a_{21}=r_{1} c+\frac{r_{2} \alpha \hat{I} \hat{S}}{\hat{N}^{2}}, \quad a_{22}=r_{2}-\frac{r_{2} \alpha \hat{I}(\hat{J}+\hat{I})}{\hat{N}^{2}}, \quad a_{23}=r_{3} \sigma-\frac{r_{2} \alpha \hat{S}(\hat{J}+\hat{S})}{\hat{N}^{2}}
\end{aligned}
$$

and

$$
a_{31}=\frac{-r_{2} \alpha \hat{I} \hat{S}}{\hat{N}^{2}}, \quad a_{32}=\frac{r_{2} \alpha \hat{I}(\hat{J}+\hat{I})}{\hat{N}^{2}}, \quad a_{33}=r_{3}(1-\sigma)+\frac{r_{2} \alpha \hat{S}(\hat{J}+\hat{S})}{\hat{N}^{2}} .
$$

The characteristic equation of $J(\hat{J}, \hat{S}, \hat{I})$ is

$$
F(\lambda)=\lambda^{3}+b_{1} \lambda^{2}+b_{2} \lambda+b_{3}=0,
$$

where

$$
\begin{aligned}
& b_{1}=-a_{11}-a_{22}-a_{33}, \\
& b_{2}=a_{11} a_{22}+a_{11} a_{33}-a_{12} a_{21}-a_{13} a_{31}+a_{22} a_{33}-a_{23} a_{32}, \\
& b_{3}=-a_{11} a_{22} a_{33}-a_{11} a_{23} a_{32}+a_{12} a_{21} a_{33}+a_{12} a_{23} a_{31}-a_{13} a_{21} a_{23}+a_{13} a_{22} a_{31} .
\end{aligned}
$$

Let

$$
A=b_{1}^{2}-3 b_{2}, \quad B=b_{1} b_{2}-9 b_{3}, \quad C=b_{2}^{2}-3 b_{1} b_{3}
$$

and

$$
\Delta=B^{2}-4 A C=-3 b_{1}^{2} b_{2}^{2}-54 b_{1} b_{2} b_{3}+81 b_{3}^{2}+12 b_{1}^{3} b_{3}+12 b_{2}^{3} .
$$


Further, it follows that the derivative of $F^{\prime}(\lambda)$ is

$$
F^{\prime}(\lambda)=3 \lambda^{2}+2 b_{1} \lambda+b_{2}
$$

It is easy to compute that equation $F^{\prime}(\lambda)=0$ has two roots as follows:

$$
\lambda_{1,2}^{*}=\frac{1}{3}\left(-b_{1} \pm \sqrt{b_{1}^{2}-3 b_{2}}\right) .
$$

When $\Delta \leq 0$, by Lemma 2.5, we have that equation (30) has three real roots $\lambda_{1}$, $\lambda_{2}$ and $\lambda_{3}$. From this, we can easily prove that two roots $\lambda_{1,2}^{*}$ of equation $F^{\prime}(\lambda)=0$ also are real.

When $\Delta>0$, by Lemma 2.5 , we have that equation (30) has one real root $\lambda_{1}$ and a pair of conjugate complex roots $\lambda_{2,3}$ and the conjugate complex roots are

$$
\lambda_{2,3}=\frac{-2 b_{1}+X_{1}^{\frac{1}{3}}+X_{2}^{\frac{1}{3}}}{6} \pm i \frac{\sqrt{3}\left(X_{1}^{\frac{1}{3}}-X_{2}^{\frac{1}{3}}\right)}{6}
$$

where

$$
X_{1,2}=b_{1} A+\frac{-B \pm \sqrt{B^{2}-4 A C}}{2} .
$$

Further, we have

$$
F(1)=1+b_{1}+b_{2}+b_{3}
$$

and

$$
F(-1)=-1+b_{1}-b_{2}+b_{3} .
$$

Therefore, on the local asymptotical stability of endemic equilibrium $(\hat{J}, \hat{S}, \hat{I})$, we have the following result.

Theorem 4.3 Suppose that $R_{0}^{*}>1$ and $R_{1}^{*}>1$. If one of the following conditions holds, then endemic equilibrium $(\hat{J}, \hat{S}, \hat{I})$ of model (1) is locally asymptotically stable.

(a) $\Delta \leq 0, F(1)>0, F(-1)<0$ and the roots $\lambda_{1,2}^{*}$ of $F^{\prime}(\lambda)=0$ satisfy $-1<\lambda_{1,2}^{*}<1$.

(b) $\Delta>0, F(1)>0, F(-1)<0$ and the conjugate complex roots $\lambda_{2,3}$ satisfy $\left|\lambda_{2,3}\right|<1$.

Proof Let $\Delta \leq 0$, then equation (30) has three real roots $\lambda_{1}, \lambda_{2}$ and $\lambda_{3}$. We assume $\lambda_{1} \leq \lambda_{2} \leq \lambda_{3}$. Further, we obtain that equation $F^{\prime}(\lambda)=0$ has two real roots $\lambda_{1}^{*}$ and $\lambda_{2}^{*}$ with $\lambda_{1}^{*} \leq \lambda_{2}^{*}$. From the expression of $F^{\prime}(\lambda)$ we have $F^{\prime}(\lambda)>0$ for all $\lambda \in\left(-\infty, \lambda_{1}^{*}\right) \cup\left(\lambda_{2}^{*},+\infty\right)$ and $F^{\prime}(\lambda)<0$ for all $\lambda \in\left(\lambda_{1}^{*}, \lambda_{2}^{*}\right)$. Hence, $F(\lambda)$ is increasing for all $\lambda \in\left(-\infty, \lambda_{1}^{*}\right) \cup\left(\lambda_{2}^{*},+\infty\right)$ and decreasing for all $\lambda \in\left(\lambda_{1}^{*}, \lambda_{2}^{*}\right)$. Therefore, we finally obtain $F\left(\lambda_{1}^{*}\right) \geq 0, F\left(\lambda_{2}^{*}\right) \leq 0, \lambda_{1} \in\left(-\infty, \lambda_{1}^{*}\right]$, $\lambda_{2} \in\left[\lambda_{1}^{*}, \lambda_{2}^{*}\right]$ and $\lambda_{3} \in\left[\lambda_{2}^{*},+\infty\right)$.

Let condition (a) hold. Then we obviously have $\lambda_{1} \in\left(-1, \lambda_{1}^{*}\right], \lambda_{2} \in\left[\lambda_{1}^{*}, \lambda_{2}^{*}\right]$ and $\lambda_{3} \in\left[\lambda_{2}^{*}, 1\right)$. Therefore, $(\hat{S}, \hat{I}, \hat{R})$ is locally asymptotically stable.

Now, we let $\Delta>0$. If condition (b) holds, then from $F(1)>0$ and $F(-1)<0$ we have that real root $\lambda_{1} \in(-1,1)$. Therefore, from $\left|\lambda_{2,3}\right|<1$ we obtain that $(\hat{S}, \hat{I}, \hat{R})$ is also locally asymptotically stable. This completes the proof of Theorem 4.3. 
Remark 4.3 From Theorem 4.3, we note that model (1) has only an endemic equilibrium and which is locally asymptotical stability. It is different from continuous epidemic models. So, discrete epidemic models have more complex dynamic behaviors than normal.

\section{Numerical simulation}

In this section, we carry out numerical simulations on model (1) to demonstrate the results in Sections 3 and 4.

Example 5.1 Consider model (1) with the following parameters:

$$
\begin{aligned}
& r_{1}=0.9, \quad r_{2}=0.95, \quad r_{3}=0.94, \quad \xi=1, \\
& \beta=15, \quad \alpha=0.4, \quad \sigma=0.001, \quad c=0.65 .
\end{aligned}
$$

By calculating, we obtain that $R_{0}^{*}=2.5858>1$ and $R_{1}^{*}=1.2803>1$. It implies that model (1) has only an endemic equilibrium. Further, by calculating we have that $R_{0}=1.0170>$ $1, \Delta=0.0363, F(1)=0.0033, F(-1)=-3.8399$ and $\lambda_{2,3}=0.8278 \pm 0.2744 \mathbf{i}$ with $\left|\lambda_{2,3}\right|=$ $0.87209<1$. Therefore, the disease in model (1) is permanent from Theorem 3.2 and the endemic equilibrium is locally asymptotically stable from Theorem 4.3. The numerical simulation is given in Figure 1.

Example 5.2 Consider model (1) with the following parameters:

$$
\begin{aligned}
& r_{1}=0.8, \quad r_{2}=0.7, \quad r_{3}=0.7, \quad \xi=1, \\
& \beta=15, \quad \alpha=0.4, \quad \sigma=0.01, \quad c=0.65 \text {. }
\end{aligned}
$$

By calculating, we have $B^{\prime \prime}=1.2452>1$ and $R_{1}=0.9730<1$. Therefore, the disease-free equilibrium of model (1) is globally stable, which implies that the disease is extinct from
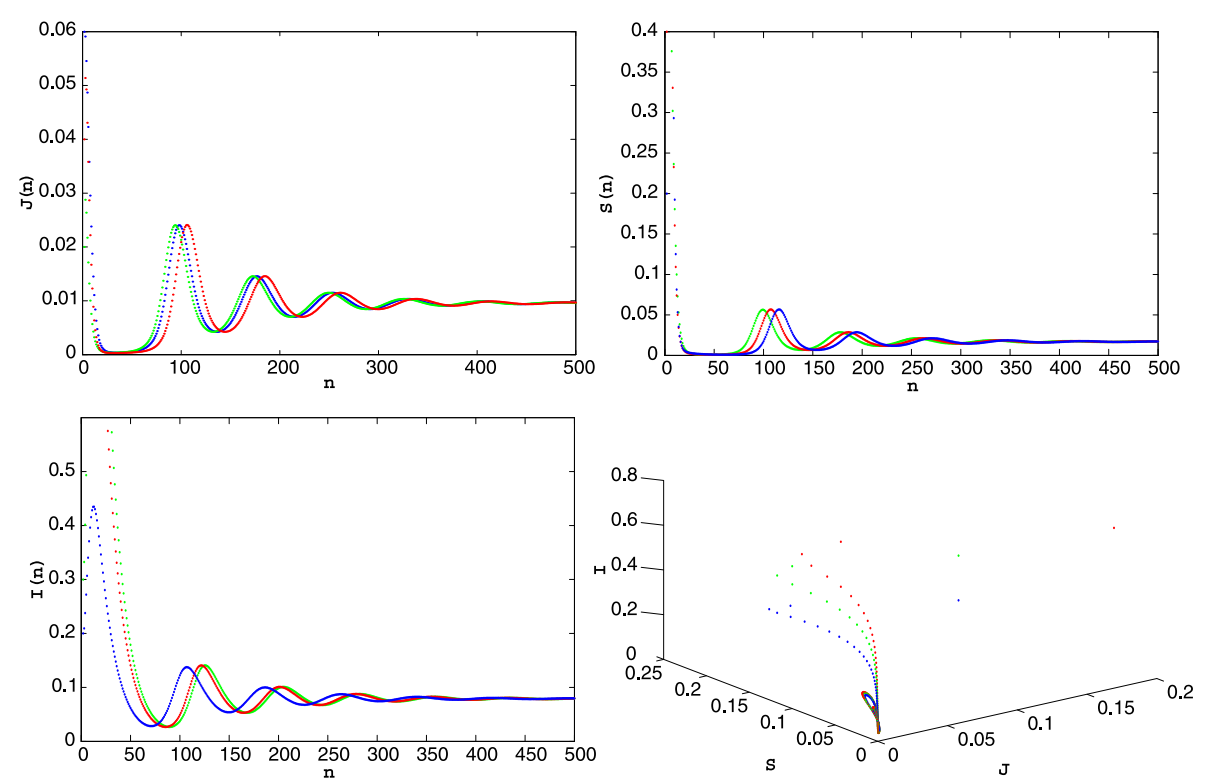

Figure 1 The permanence of infected mature population with different initial values. 


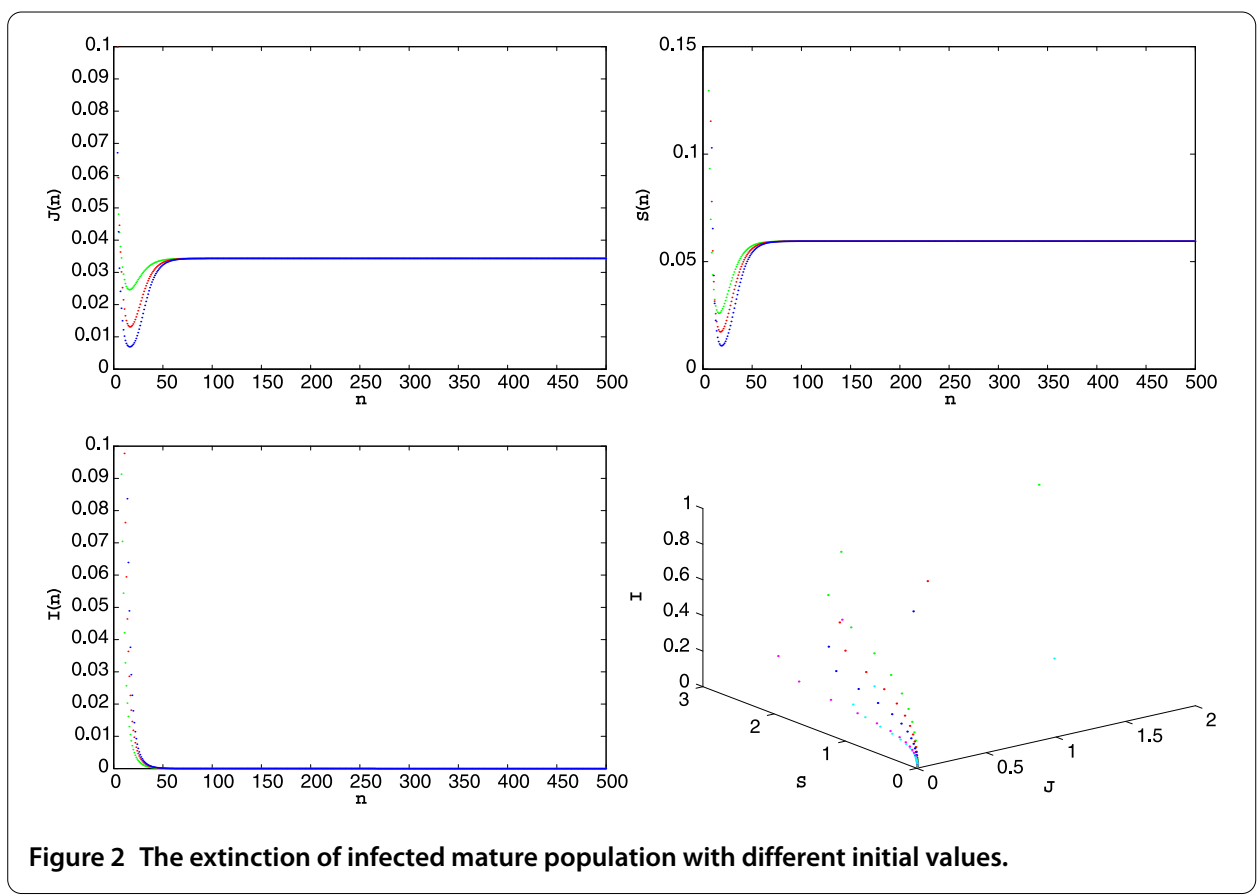

Theorem 3.3. The numerical simulation is given in Figure 2, which shown that the mature infected individuals is extinct.

Finally, we easily see that model (1) can exhibit more complicated dynamical behaviors from the following two examples.

Example 5.3 Consider model (1) with the following parameters:

$$
\begin{array}{ll}
r_{1}=0.8, & r_{2}=0.95, \quad r_{3}=0.95, \quad \xi=1, \quad \beta=0.5, \\
\alpha=0.7, \quad \sigma=0.001, & 0.4 \leq c \leq 0.65 .
\end{array}
$$

By carefully calculating, we can obtain

$$
R_{0}=0.0046 S^{*}+0.949, \quad B^{*}=\frac{11.189 c}{1+4 c}
$$

where

$$
S^{*}=\frac{32 c(76 c-1)}{(1+4 c)(1+16 c)} .
$$

From the expressions of $R_{0}$ and $S^{\prime \prime}$, we easily obtain $R_{0}>1$ for any $c \geq 0.4$. Which implies that model (1) is permanent and has at least endemic equilibrium. However, the numerical simulation shows that the bifurcation appears on parameter $c \in[0.4,0.65]$ in Figure 3.

Particularly, take $c=0.44$, by calculating we obtain $R_{0}=1.0438>1, R_{0}^{*}=1.1159>1$ and $R_{1}^{*}=1.5214>1$. Further, $\Delta=0.0869>0, F(-1)=-3.4377<0, F(1)=0.0048>0, \lambda_{2,3}=$ $0.2773 \pm 0.0268 \mathbf{i}$. So, by Theorems 4.2 and 4.3 , model (1) has only an endemic equilibrium and which is locally asymptotically stable. At same time, the numerical simulation shows that the solutions of model (1) converge to a periodic cycle in Figure 4. 

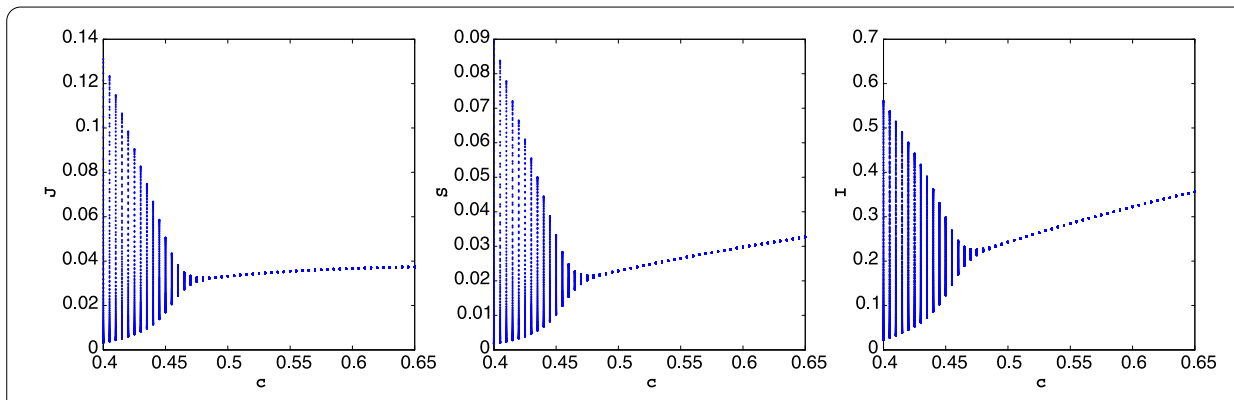

Figure 3 The bifurcation of infected mature population with $0.4 \leq c \leq 0.65$.

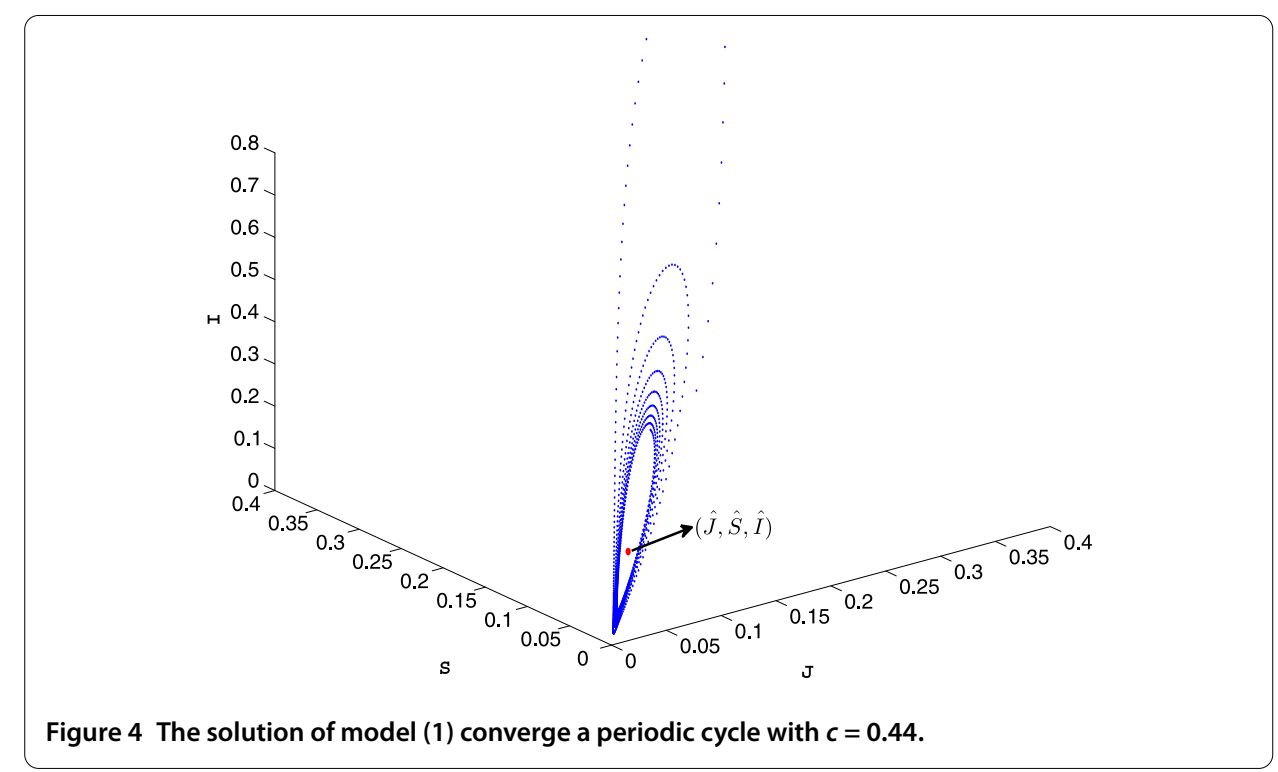

Example 5.4 We investigate model (1) with the parameters as follows:

$$
\begin{aligned}
& r_{2}=0.95, \quad r_{3}=0.95, \quad \xi=1, \quad \beta=0.5, \quad \alpha=0.7, \\
& \sigma=0.001, \quad c=0.4, \quad 0.78 \leq r_{1} \leq 0.9 .
\end{aligned}
$$

By carefully calculating, we can obtain

$$
R_{0}=0.0046 S^{*}+0.949, \quad B^{*}=\frac{2.7972 r_{1}}{5-3 r_{1}}
$$

where

$$
S^{*}=\frac{16 r_{1}\left(43 r_{1}-5\right)}{\left(5-3 r_{1}\right)\left(1+8 r_{1}\right)}
$$

From the expressions of $R_{0}, S^{*}$ and $B^{*}$, we easily obtain $R_{0}>1$ for any $r_{1} \in[0.7,0.9]$ and $B^{*}>1$ for any $r_{1} \in[0.86,0.9]$. The numerical simulation on parameter $r_{1} \in[0.78,0.9]$ is given in Figure 5. 

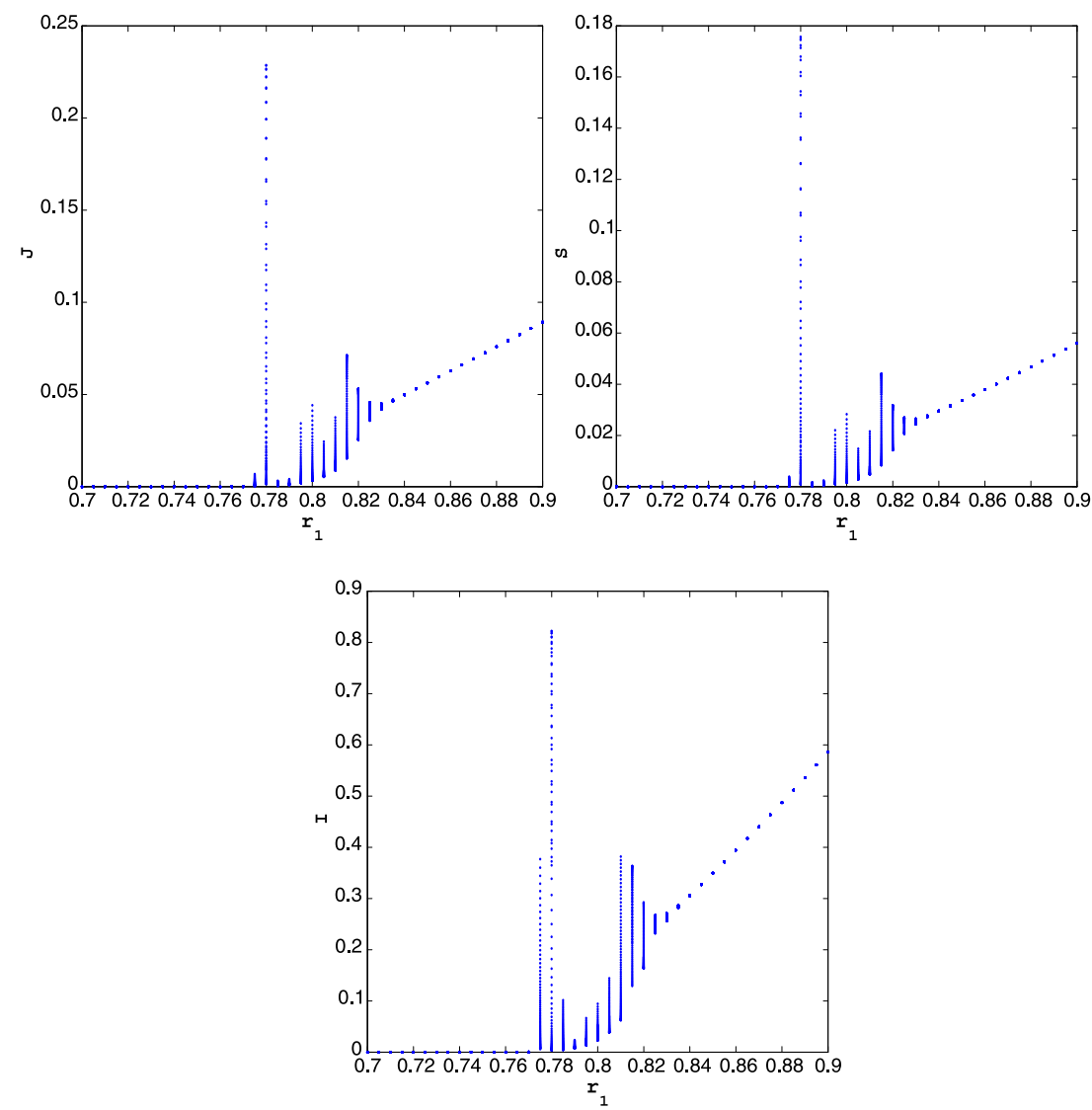

Figure 5 The bifurcation of infected mature population with $0.7 \leq r_{1} \leq 0.9$.

From Figure 5, we can see that the disease free equilibrium is locally asymptotically stable when $r_{1} \in(0,0.77)$, the endemic equilibrium is locally asymptotically stable when $r_{1} \in(0.843,0.9)$ and the bifurcation appears when $r_{1} \in(0.777,0.843)$.

Particularly, take $r_{1}=0.8$, by calculating we obtain $R_{0}=1.0391>1, R_{0}^{*}=1.0903>1$ and $R_{1}^{*}=1.5144>1$. Further, $\Delta=0.0875>0, F(-1)=-3.5940<0, F(1)=0.0053>0, \lambda_{2,3}=$ $0.2936 \pm 0.0274$ i. So, by Theorems 4.2 and 4.3 , model (1) has only an endemic equilibrium and which is locally asymptotically stable. At same time, the numerical simulation shows that the solutions of model (1) converge to a periodic cycle in Figure 6.

\section{Discussion}

In this paper, we discuss the dynamical behaviors of model (1). In order to the sufficient conditions of the permanence and distinct of the disease, we first establish some lemmas for disease free model (3) and auxiliary system (7) in Section 2. From the results of Theorems 3.2 and 3.3, we can see that there exists a gap between $R_{0}$ and $R_{1}$. Furthermore, the existence and local stability of the endemic equilibrium are discussed. In view of the numerical simulations, we note that the discrete epidemic models have more complex dynamic behaviors than the corresponding continuous epidemic models.

In model (1), standard incidence is considered, however saturated incidence $g(I)=$ $k I /(1+\alpha I)$ rate may be more realistic than standard incidence. Therefore, we propose the 


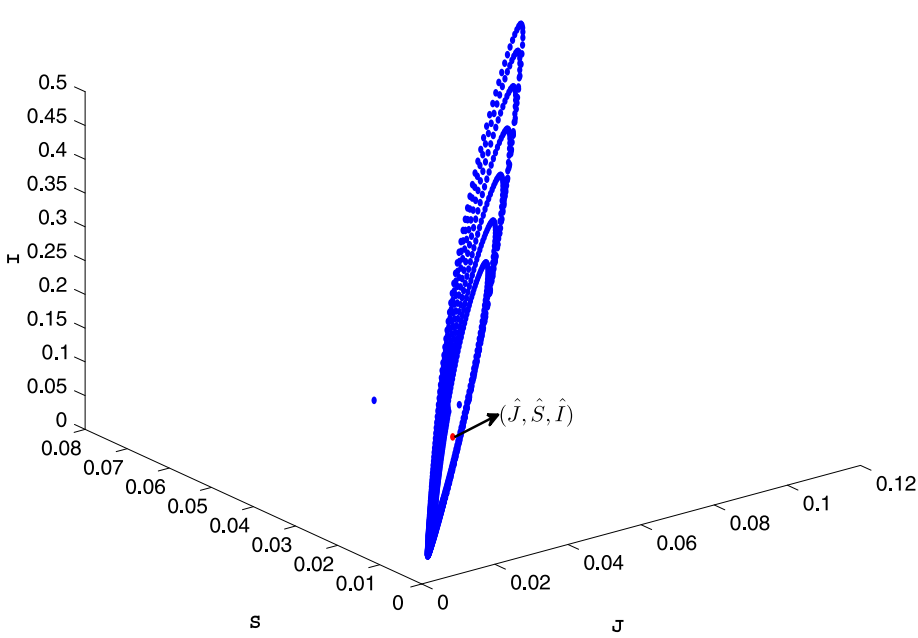

Figure 6 The solution of model (1) converge a periodic cycle with $r_{1}=0.8$.

following epidemic model in discrete time with stage structure:

$$
\left\{\begin{array}{l}
J(n+1)=\frac{\xi S(n)}{1+\beta N(n)}+r_{1} J(n)-r_{1} c J(n), \\
S(n+1)=r_{1} c J(n)+r_{2}\left(1-\frac{k I(n)}{1+\alpha I(n)}\right) S(n)+r_{3} \sigma I(n), \\
I(n+1)=r_{2} \frac{k I(n)}{1+\alpha I(n)} S(n)+r_{3}(1-\sigma) I(n), \\
N(n)=J(n)+S(n)+I(n) .
\end{array}\right.
$$

Further, we introduce more general incidence $g(I)=\frac{k l^{q}}{1+\alpha I^{p}}$ which was proposed by Liu in [4], here $q$ and $p$ are positive integers. We can obtain the following model:

$$
\left\{\begin{array}{l}
J(n+1)=\frac{\xi S(n)}{1+\beta N(n)}+r_{1} J(n)-r_{1} c J(n), \\
S(n+1)=r_{1} c J(n)+r_{2}\left(1-\frac{k I^{q}(n)}{1+\alpha I^{p}(n)}\right) S(n)+r_{3} \sigma I(n), \\
I(n+1)=r_{2} \frac{k I^{q}(n)}{1+\alpha I^{p}(n)} S(n)+r_{3}(1-\sigma) I(n), \\
N(n)=J(n)+S(n)+I(n) .
\end{array}\right.
$$

We will discuss these models in our future works.

\section{Competing interests}

The authors declare that they have no competing interests.

\section{Authors' contributions}

The authors declare that the study was realized in collaboration with the same responsibility. All authors read and approved the final manuscript.

\section{Acknowledgements}

This work is in part supported by the Scientific Research Programmes of Colleges in Xinjiang (Grant No. XJEDU2011S08), the National Natural Science Foundation of China (Grant Nos. 11271312, 11001235), the China Postdoctoral Science Foundation (Grant Nos. 20110491750, 2012T50836) and the Natural Science Foundation of Xinjiang (Grant No. 2011211B08)

Received: 30 September 2012 Accepted: 15 March 2013 Published: 3 April 2013

\section{References}

1. Castillo-Chavez, C, Yakubu, A-A: Discrete-time SIS models with complex dynamics. Nonlinear Anal. 47, 4753-4762 (2001) 
2. Allen, LJS: Some discrete-time SI, SIR, and SIS epidemic models. Math. Biosci. 124, 83-105 (1994)

3. Li, X, Wang, W: A discrete epidemic model with stage structure. Chaos Solitons Fractals 26, 947-958 (2005)

4. Li, J, Ma, ZE, Brauer, F: Global analysis of discrete-time SI and SIS epidemic models. Math. Biosci. Eng. 4, 699-710 (2007)

5. Ramani, A, Carstea, A, Willox, R, Grammaticos, B: Oscillating epidemics: a discrete-time model. Physica A 333, 278-292 (2004)

6. Satsuma, J, Willox, R, Ramani, A, Grammaticos, B, Carstea, A: Extending the SIR epidemic model. Physica A 336 369-375 (2004)

7. Zhang, D, Shi, B: Oscillation and global asymptotic stability in a discrete epidemic model. J. Math. Anal. Appl. 278, 194-202 (2003)

8. Mendez, V, Fort, J: Dynamical evolution of discrete epidemic models. Physica A 284, 309-317 (2000)

9. Zhou, YC, Ma, EN, Brauer, F: A discrete epidemic model for SARS transmission and control in China. Math. Comput. Model. 40, 1491-1506 (2004)

10. Allen, LJS, Driessche, P: The basic reproduction number in some discrete-time epidemic models. J. Differ. Equ. Appl. 14, 1127-1147 (2008)

11. D'innocenzo, A, Paladini, F, Renna, L: A numerical investigation of discrete oscillating epidemic models. Physica A 364 , 497-512 (2006)

12. Willoxa, R, Grammaticosa, B, Carsteab, AS, Ramani, A: Epidemic dynamics: discrete-time and cellular automaton models. Physica A 328, 13-22 (2003)

13. Franke, JE, Yakubu, A-A: Disease-induced mortality in density-dependent discrete-time S-I-S epidemic models. J. Math. Biol. 57, 755-790 (2008)

14. Allen, LJS, Lou, Y, Nevai, AL: Spatial patterns in a discrete-time SIS patch model. J. Math. Biol. 58, 339-375 (2009)

15. Li, J, Lou, J, Lou, M: Some discrete SI and SIS epidemic models. Appl. Math. Mech. 29, 113-119 (2008)

16. Zhou, Y, Pergola, P: Dynamics of a discrete age-structure SIS models. Discrete Contin. Dyn. Syst., Ser. B 4, 841-850 (2004)

17. Anderson, R, May, R: Infectious Diseases of Human: Dynamics and Control. Oxford University Press, Oxford (1991)

18. Capsso, V, Serio, G: A generalization of the Kermack-Mckendrick deterministic epidemic model. Math. Biosci. 42, 43-61 (1978)

19. Liu, W, Levin, SA, Iwasa, Y: Influence of nonlinear incidence rates upon the behavior of SIRS epidemiological models. J. Math. Biol. 23, 187-204 (1986)

20. Xiao, YN, Chen, LS: On an SIS epidemic model with stage structure. J. Syst. Sci. Complex. 2, 275-288 (2003)

21. Wang, L, Wang, MQ: Ordinary Difference Equation. Xinjiang Univ. Press, Xinjiang (1989) (in Chinese)

22. Fan, S: A new extracting formula and a new distinguishing means on the one variable cubic equation. Nat. Sci. J. Hainan Teach. Coll. 2, 91-98 (1989)

doi:10.1186/1687-1847-2013-87

Cite this article as: Teng et al.: Dynamical behaviors of a discrete SIS epidemic model with standard incidence and stage structure. Advances in Difference Equations 2013 2013:87.

\section{Submit your manuscript to a SpringerOpen ${ }^{\circ}$ journal and benefit from:}

- Convenient online submission

Rigorous peer review

- Immediate publication on acceptance

- Open access: articles freely available online

- High visibility within the field

- Retaining the copyright to your article 